\title{
Measurement of DNA repair activity in hepatocytes exposed to fatty acids
}

\author{
E. Healing ${ }^{1}$, L.B. Meira ${ }^{1}$, P.J. Aston ${ }^{2}$, M.J. Tindall ${ }^{3}$ and R.M. Elliott ${ }^{1}$ \\ ${ }^{1}$ School of Biosciences, Faculty of Health and Medical Sciences, ${ }^{2}$ Department of Mathematics, Faculty of Engineering \\ and Physical Sciences, University of Surrey, GU2 7XH and ${ }^{3}$ Department of Mathematics and Statistics and Institute \\ for Cardiovascular and Metabolic Research, Faculty of Science, University of Reading, RG6 7BE
}

DNA repair capacity varies greatly between individuals ${ }^{(1)}$, and evidence has begun to link this variation to cancer risk, obesity and related chronic diseases ${ }^{(2)}$. There is also emerging evidence that dietary components can affect DNA repair ${ }^{(3)}$, but research to date has been restricted by methods for measuring DNA repair ${ }^{(4)}$. This study made use of newly developed microplate-based assays for the direct determination of DNA repair enzyme activities. Lipid loading of the HepG2 human hepatocellular carcinoma cell line was employed as a model to test the hypothesis that hepatic steatosis affects DNA repair activity via induction of oxidative stress.

HepG2 cells were treated with palmitic acid (PA), oleic acid (OA) or vehicle for 24 hours before determining cell viability, intracellular lipid levels, and DNA repair enzyme activity (uracil DNA glycosylase (hUNG1) and 8-oxoguanine DNA glycosylase (hOGG1)). For the repair assays, oligonucleotides designed to form a hairpin loop with a $5^{\prime}$ fluorescein and a damaged base (uracil for hUNG1 assay or 8-oxoguanine for hOGG1 assay) within the double stranded region were bound to the surface of Nunc Immobiliser ${ }^{\mathrm{TM}}$ plates via a $3^{\prime}$ amino group. HepG2 nuclear extract or recombinant enzyme was incubated with each substrate, creating alkali-labile abasic sites (hUNG1) or single strand breaks (hOGG1). Following alkaline denaturation, enzyme activity was determined by quantifying the retained fluorescein.

Treatment with $400 \mu \mathrm{M}$ PA decreased cell viability to $61 \%(P<0.01$ compared to vehicle) (Fig. 1A.). Intracellular lipid was significantly increased following treatment with $300 \mu \mathrm{M}$ and $400 \mu \mathrm{M} \mathrm{OA}(P<0.05$ and $P<0 \cdot 001$, respectively), and $300 \mu \mathrm{M}$ and $400 \mu \mathrm{M}$ PA $(P<0.001$ and $P<0.0001$, respectively) compared to vehicle (Fig. 1B.). No differences in hUNG1 or hOGG1 activity were detected between treatment groups (Fig. 1C.).
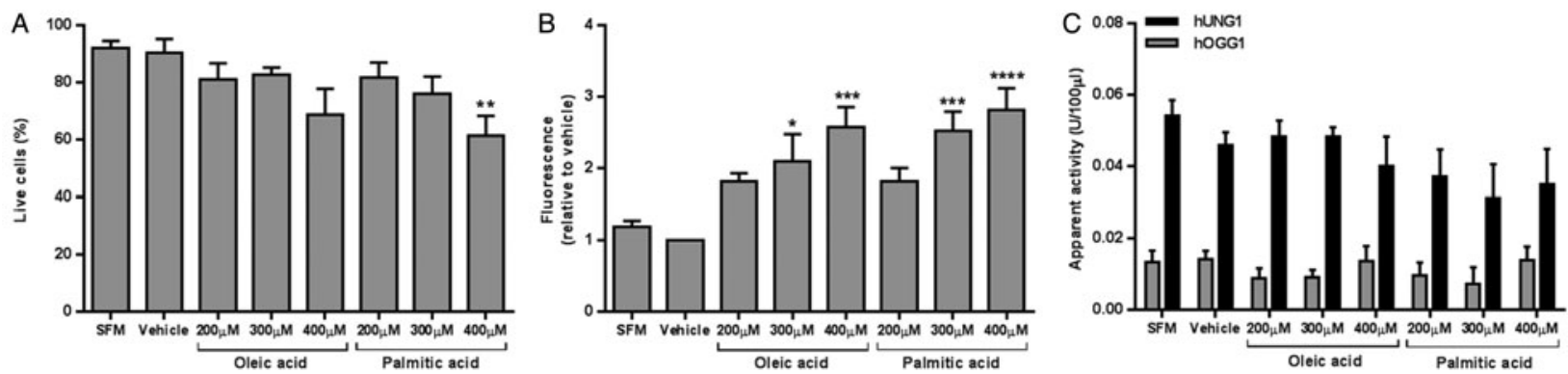

Fig. 1. HepG2 viability (A), intracellular lipid level (B), and apparent DNA glycosylase activity (C) following 24 hours of growth with SFM, vehicle, or fatty acid. Data presented as mean + SEM of 6 independent experiments. SFM: serum free media; hUNG1: uracil DNA glycosylase; hOGG1: 8-oxoguanine DNA glycosylase. ${ }^{*} P<0.05,{ }^{*} P<0.01,{ }^{*} * * P<0.001, * * * * P<0.0001$ compared to vehicle (ANOVA, followed by Sidak's test).

In conclusion, fatty acid treatment reduced cell viability and increased intracellular lipid levels in a dose-dependent manner. The use of novel assays enabled quantitative determination of DNA repair enzyme activities in cell extracts. The absence of detectable effects of lipid loading on DNA repair could be due to the use of cancer cells as a model, which may be lacking certain DNA repair regulatory mechanisms normally present in healthy cells. Future work will focus on optimisation of the assays for use with primary cells samples from human volunteers.

This work was funded by a studentship awarded by the Biotechnology and Biological Sciences Research Council Doctoral Training Programme.

1. Wilson D, Kim D, Berquist B et al. (2011) Mut Res 711, 100-12

2. Sampath H, Vartanian V, Rollins R et al. (2012) PLoS One 7, e51697

3. Collins A, Azqueta A \& Langie S (2012) Eur J Nutr 51, 261-279

4. Nagel Z, Chaim I \& Samson L (2014) DNA Repair (Amst) 19, 199-213 